\title{
Comparision of Direct Antibiotic Susceptibility Testing with Standard Testing in Blood Culture
}

\author{
Rahila Yakoob* and Gopalkrishna Bhat K. \\ Department of Microbiology, Kasturba Medical College, Mangalore - 575 003, India.
}

\begin{abstract}
To perform Direct Antibiotic Susceptibility Testing (DAST) on positive blood culture and compare with that of standard antibiotic susceptibility testing. Blood cultures performed at Department of Microbiology Kasturba Medical College and Microbiology diagnostic centre (KMC hospital Ambedkar circle), Mangalore. Blood cultures with monobacterial bacteraemia. Cross sectional comparative time bound study of 116 samples. Out of 116 positive blood culture samples. It was seen that over all error rate was $55.17 \%$ minor error/categorical error, $15.5 \%$ of major error and $0.8 \%$ of very major errors were detected. The inoculum size influences the result of DAST and standard AST. Therefore standardization of inoculum is crucial. To conclude DAST using disk diffusion from positive blood culture bottles help to start early antibiotic treatment for bacteraemia/septicaemia.
\end{abstract}

Keywords: Blood stream infection, Direct antibiotic susceptibility testing, concordance.

*Correspondence: rahilayakub@gmail.com; +91-9633018152

(Received: 09 March 2018; accepted: 26 May 2018)

Citation: Rahila Yakoob and Gopalkrishna Bhat K., Comparision of Direct Antibiotic Susceptibility Testing with Standard Testing in Blood Culture, J Pure Appl Microbiol., 2018; 12(4):2289-2296. http://dx.doi.org/10.22207/JPAM.12.4.70

(c) The Author(s) 2018. Open Access. This article is distributed under the terms of the Creative Commons Attribution 4.0 International License which permits unrestricted use, sharing, distribution, and reproduction in any medium, provided you give appropriate credit to the original author(s) and the source, provide a link to the Creative Commons license, and indicate if changes were made. 


\section{INTRODUCTION}

Sepsis is one of the leading causes of morbidity and mortality in hospitalised patients ${ }^{1}$. Accurate information of patients with blood stream infections can be obtained by performing antibiotic susceptibility test and identification of bacteria ${ }^{2}$. Timely and early information regarding the identification and susceptibility pattern of significant bacteria helps the clinicians in rapid diagnosis, determine resistance pattern both in community and institutions and also contribute to the reduction in hospital-care associated costs ${ }^{3}$. Appropriate treatment and early diagnosis of blood stream infection can save the life of people ${ }^{4}$.

The conventional phenotypic methods, based on culturing on agar (e.g.: Disk diffusion test) or on micro titration plates (e.g.: broth dilution method) is one of the commonly employed methods ${ }^{2}$. In case of standard AST, the results are available only with a delay of $48-72 \mathrm{~h}$ after sampling as bacteria needed to be cultured before AST can be executed ${ }^{2}$. Standardised of inoculum is a main problem in DAST, so it has been criticised by American Society for Microbiology (ASM), the British Society for Antimicrobial Chemotherapy (BASC), and the European Committee on Antimicrobial Susceptibility Testing (EUCAST) ${ }^{2}$.

Disk diffusion has many advantages such as it allows the visibility of growth, correct inoculum, mixed cultures and other abnormalities and it is flexible and cheap. And another benefit is the possibility of executing DAST ${ }^{2}$. Rapid and most reliable results for direct and standard inoculation are provided by the non-fastidious Enterobacteriaceae family among all the other bacteria $^{5}$. DAST by turbid broth is an accepted method for rapid reporting. Although not mentioned in CLSI, but standardization of broth for direct sensitivity is mentioned in BSAC (British Society for Antimicrobial Chemotherapy) methods ${ }^{6,7}$. Previous studies comparing DAST with conventional method have yielded varying results and these studies were conducted only on gram negative bacilli. The study focuses on performance and clinical significance of DAST in blood culture.

\section{MATERIALS AND METHODS}

The study was conducted at the Department of Microbiology, Kasturba Medical College, Mangalore and blood samples for culture were received from District Wenlock Hospital, Lady Goschen Hospital and Kasturba Medical College Hospital, Ambedkar Circle, Mangalore.

\section{Study duration}

The study was conducted from January 2017 to June 2017.

\section{Study design}

Cross sectional comparative time bound study.

Sample size

A total of 116 positive blood culture samples were included in the study (time bound). Inclusion criteria

Blood cultures with monobacterial bacteraemia.

\section{Exclusion criteria}

1) Blood cultures with two or more isolates. 2) Blood cultures positive for fungi.

\section{Ethical consideration}

The present study had approval of the Institutional Ethics Committee (IEC KMC MLR 11$16 / 305,16^{\text {th }}$ November 2016 ).

\section{Processing of samples}

Samples of blood was collected with aseptic precaution from patients with suspected bacteraemia/septicaemia and was inoculated into blood culture bottles and incubated in BACTEC 9050 or BacT/ALERT systems. When the system beeps showing growth of bacteria in blood culture bottles, bottle was removed and an aliquot of sample was used for smear preparation and Gram staining. Samples which show single type of bacteria were used for the study.

Direct Antibiotic Susceptibility Testing (DAST) By Disk Diffusion Method

\section{Gram negative bacilli}

A drop $(20 \mu \mathrm{l})$ of blood was placed in $5 \mathrm{ml}$ of sterile water using the venting needle. A sterile cotton wool swab was dipped and excess was removed by turning the swab against the walls of the container. The swab was used to spread the inoculum evenly over the surface of susceptibility plate (6).The following panels of antimicrobial disks were used: amikacin $(30 \mu \mathrm{g})$, ampicillin $(10 \mu \mathrm{g})$, amoxicillin/ clavulanic acid $(20 / 10 \mu \mathrm{g})$,cefuroxime $(30 \mu \mathrm{g})$, cefepime $(30 \mu \mathrm{g})$, ceftriaxone $(30 \mu \mathrm{g})$, ciprofloxacin $(5 \mu \mathrm{g})$, cefoperazone/sulbactum $(5 \mu \mathrm{g})$, trimethoprim/sulfamethoxazole $(1.25 / 23.75 \mu \mathrm{g})$, ertapenem $(10 \mu \mathrm{g})$, gentamicin $(10 \mu \mathrm{g})$, imipenem 
$(10 \mu \mathrm{g})$, meropenem $(10 \mu \mathrm{g})$, piperacillin/ tazobactum $(10 \mu \mathrm{g})$, tigecycline $(15 \mu \mathrm{g})$. The zone inhibition were interpreted as Susceptibile (S), Intermediate (I), Resistant (R) as per CLSI guidelines 2016[9].

\section{Gram positive cocci}

Three drops $(60 \mu \mathrm{l})$ of blood was mixed in $5 \mathrm{ml}$ of sterile water using the venting needle. A sterile cotton wool swab was dipped and excess was removed by turning the swab against the walls of the container. The swab was then used to spread the inoculum evenly over the surface of susceptibility plate (1). The following panels of antimicrobial disks were used; For Staphylococcus aureus and coagulase negative staphylococci: clindamycin $(2 \mu \mathrm{g})$, cefoxitin $(30 \mu \mathrm{g})$ ciprofloxacin $(5 \mu \mathrm{g})$,erythromycin $(5 \mu \mathrm{g})$ gentamicin $(10 \mu \mathrm{g})$, linezolid $(30 \mu \mathrm{g})$, rifampicin $(5 \mu \mathrm{g})$, penicillin (10units), trimethoprim/sulfamethaxazole $(1.25 / 3.75 \mu \mathrm{g})$, teicoplanin $(30 \mu \mathrm{g})$.

For enterococcus species: Ampicillin (10 $\mu \mathrm{g})$, amikacin $(30 \mu \mathrm{g})$, high level gentamicin (120 $\mu \mathrm{g})$, high level streptomycin $(300 \mu \mathrm{g})$,imipenem $(10 \mu \mathrm{g})$,meropenem $(10 \mu \mathrm{g})$, pencillin (10units), teicoplanin $(30 \mu \mathrm{g})$ and vancomycin $(30 \mu \mathrm{g})$. The zone inhibition were interpreted as Susceptibile(S), Intermediate (I), Resistant (R) as per CLSI guidelines $2016^{[9] .}$. Each bottle positive flagged by the BACTEC or BacT/ALERT instrument was inoculated on Mac Conkey agar, blood agar and chocolate agar and was incubated at $37^{\circ} \mathrm{C}$ for 24 hours. Standard antibiotic susceptibility testing was done using the culture isolates, by Kirby Bauer disk diffusion method or VITEK 2 system ${ }^{1,8}$.

\section{Conventional susceptibility testing}

An aliquot of the positive blood culture broth was plated on Mac Conkey Agar, Blood Agar and chocolate agar which were procured from HiMedia Laboratories Limited, Mumbai, India, and incubated at $35^{\circ} \mathrm{C}$ overnight to obtain isolated colonies. These colonies will be inoculated to Muller-Hinton Broth to make a suspension equivalent to a 0.5 McFarland standard ${ }^{8}$. The above mentioned antibiotics were procured from HiMedia Laboratories Limited, Mumbai, India and interpreted accordingly, (CLSI guidelines 2016) .

\section{Quality control}

The quality control strains, E.coli ATCC 25922, S. aureus ATCC 25923, E.faecalis 29212 and P.aeruginosa ATCC 27853 were tested weekly by the reference method ${ }^{1}$.

\section{Interpretation of results and data analysis}

Susceptibility results obtained by direct antibiotic susceptibility testing were compared with conventional susceptibility test.

The following definitions were used:

1) Essential agreement or minor errors: standard method is susceptible (S) or resistant (R) and DAST is intermediate (I); alternatively, standard method is intermediate (I) and DAST is susceptible $(\mathrm{S})$ or resistance $(\mathrm{R})$.

2) Major errors; standard method yields susceptible (S) result whereas DAST yields resistance ( $\mathrm{R}$ )

3) Very major errors: standard method is resistance ( $R$ ) and DAST yields susceptible (S) [1].

\section{RESULTS}

A total of 116 gram negative and gram positive bacterial isolates were obtained. The distribution of gram negative bacilli and gram positive cocci is given in fig 1 .

The most frequent isolates among gram negative bacilli and gram positive isolates is given in table 1 and table 2 respectively.

It was observed that out of 29 E.coli isolates 26(93.10\%) were susceptibile to tigecycline, 24 (86.2\%) to ertapenem and meropenem. Among 23 k.pneumoniae isolates $23(100 \%)$ was susceptibile to tigecycline,19(82.6\%) to meropenem and imepenem. All the strains of S.aureus (25)isolated were found to be resistant to $25(100 \%)$ pencillin.

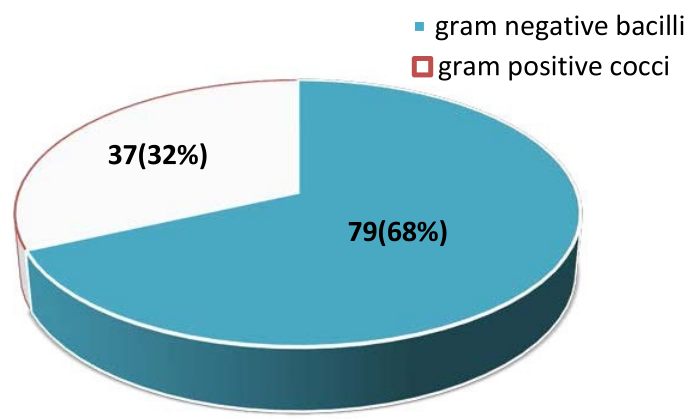

Fig. 1. Isolation of bacteria from blood culture 
Table 1. Total number of gram negative isolates from blood culture $(n=79)$

\begin{tabular}{ll}
\hline Gram negative organisms & No. (\%)isolated \\
\hline Escherichia coli & $29(25 \%)$ \\
Klebsiella pneumoniae & $23(19.82 \%)$ \\
Pseudomonas aeruginosa & $7(6.03 \%)$ \\
Salmonella typhi & $5(4.3 \%)$ \\
Acinitobacter baumannii & $3(2.58 \%)$ \\
Klebsiella oxytoca & $2(1.72 \%)$ \\
Enterobacter cloacae & $2(1.72 \%)$ \\
Citrobacter freudii & $2(1.72 \%)$ \\
Aeromonas hydrophila & $1(0.86 \%)$ \\
Proteus mirabilis & $1(0.86 \%)$ \\
Achromabacter xylosoxidans & $1(0.86 \%)$ \\
Ralstonia picketti & $10.86 \%)$ \\
\hline
\end{tabular}

Table 2. Total number of gram positive isolates from blood culture $(n=37)$

\begin{tabular}{ll}
\hline Gram positive organisms & No. (\%) isolated \\
\hline $\begin{array}{l}\text { Staphylococcus aureus } \\
\text { Coagulase negative }\end{array}$ & $25(21.5 \%)$ \\
Staphylococcus & $7(6.3 \%)$ \\
Enterococcus faecalis & $3(2.58 \%)$ \\
Enterococcus faecium & $2(1.72 \%)$ \\
\hline
\end{tabular}

For all 116 gram negative isolates; we observed $94.3 \%$ of no errors. DST yields $55.17 \%$ categorical agreement / minor error, $15.5 \%$ major error and $0.8 \%$ very major error. Direct antimicrobial susceptibility correlation for gram negative bacteria and gram positive bacteria is given in table 3,4,5,6 and 7 respectively.

\section{DISCUSSION}

Although there are many advances and diagnosis for the treatment of sepsis. Sepsis remains a major cause of death these days. Early detection of pathogen and their susceptibility pattern, plays a vital role in the diagnosis of sepsis. Immediate administration of antibiotics in sepsis is essential so as to reduce the morbidity and mortality rate in the hospitals. Mixed cultures and improper standardisation of the inoculums are the two major issues stated in the previous studies ${ }^{10}$. In the present study 116 positive blood culture samples were evaluated which were suspicious cases of septicaemia. Only unimicrobial culture was included in our study. Polymicrobial cultures were avoided based on gram stain results but few specimen that appeared to be unimicrobial were later found to produce polymicrobial growth in

Table 3. Direct antibiotic susceptibility correlation for gram negative bacilli $(n=79)$

\begin{tabular}{|c|c|c|c|c|c|c|c|c|c|}
\hline \multirow[t]{3}{*}{ Antibiotics used } & \multicolumn{9}{|c|}{ Direct susceptibility method } \\
\hline & \multicolumn{2}{|c|}{$\begin{array}{l}\text { Very major } \\
\text { error }\end{array}$} & \multicolumn{2}{|c|}{ Major error } & \multicolumn{2}{|c|}{$\begin{array}{l}\text { Minor error/ } \\
\text { essential } \\
\text { agreement }\end{array}$} & \multicolumn{3}{|c|}{ Concordance } \\
\hline & NO. & $\%$ & NO. & $\%$ & & $\%$ & & & $\%$ \\
\hline Amikacin $(30 \mu \mathrm{g})$ & 1 & 1.26 & 6 & 7.59 & 12 & 15.18 & 60 & 75 & \\
\hline Amoxicillin/Clavulanic Acid(10 $\mu \mathrm{g})$ & 1 & 1.2 & 5 & 6.3 & 7 & 8.8 & 66 & 83 & \\
\hline Ampicillin $(10 \mu \mathrm{g})$ & 0 & 0 & 0 & 0 & 0 & 0 & 79 & 1 & \\
\hline Ceftriaxone $(30 \mu \mathrm{g})$ & 0 & 0 & 0 & 0 & 0 & 0 & 79 & 1 & \\
\hline Cefuroxime $(30 \mu \mathrm{g})$ & 0 & 0 & 0 & 0 & 0 & 0 & 79 & 1 & \\
\hline Cefoperazone/Sulbactum(5 $\mu \mathrm{g})$ & 0 & 0 & 0 & 0 & 1 & 1.2 & 78 & $9 \varepsilon$ & \\
\hline Ciprofloxacin(5 $\mu \mathrm{g})$ & 0 & 0 & 1 & 1.2 & 1 & 1.2 & 77 & 97 & \\
\hline Ertapenem(10 $\mu \mathrm{g})$ & 0 & 0 & 0 & 0 & 2 & 2.5 & 77 & & \\
\hline Gentamicin $(10 \mu \mathrm{g})$ & 0 & 0 & 0 & 0 & 7 & 8.8 & 72 & 9 & \\
\hline Imipenem $(10 \mu \mathrm{g})$ & 0 & 0 & 0 & 0 & 1 & 1.2 & 78 & $9 \varepsilon$ & \\
\hline Meropenem $(10 \mu \mathrm{g})$ & 0 & 0 & 0 & 0 & 0 & 0 & 79 & & \\
\hline Piperacillin/Tazobactum(10 $\mu \mathrm{g})$ & 0 & 0 & 0 & 1.8 & 7 & 8.8 & 72 & & \\
\hline Tigecycline(15 $\mu g)$ & 0 & 0 & 6 & 7.5 & 6 & 7.5 & 73 & & \\
\hline Cefepime(30 $\mu \mathrm{g})$ & 0 & 0 & 0 & 0 & 10 & 12.6 & 69 & 87 & \\
\hline $\begin{array}{l}\text { Trimethoprim/Sulfamethoxazole } \\
(1.25 / 23.75 \mu \mathrm{g})\end{array}$ & 1 & 1.2 & 0 & 0 & 0 & 0 & 78 & & \\
\hline
\end{tabular}


Table 4. Direct antibiotic susceptibility correlation for E.coli $(n=29)$

\begin{tabular}{|c|c|c|c|c|c|c|c|c|}
\hline \multirow[t]{3}{*}{ Antibiotics used } & \multicolumn{8}{|c|}{ Direct susceptibility method } \\
\hline & \multicolumn{2}{|c|}{$\begin{array}{l}\text { Very major } \\
\text { error }\end{array}$} & \multicolumn{2}{|c|}{ Major error } & \multicolumn{2}{|c|}{$\begin{array}{l}\text { Minor error/ } \\
\text { essential } \\
\text { agreement }\end{array}$} & \multicolumn{2}{|c|}{ Concordance } \\
\hline & NO. & $\%$ & NO. & $\%$ & NO. & $\%$ & NO. & $\%$ \\
\hline Amikacin $(30 \mu \mathrm{g})$ & 1 & 3.4 & 6 & 20.6 & 6 & 20.6 & 16 & 55.1 \\
\hline Amoxicillin/Clavulanic Acid(10 $10 \mathrm{~g})$ & 0 & 0 & 5 & 17.2 & 5 & 17.2 & 19 & 65.5 \\
\hline Ampicillin(10 $\mu \mathrm{g})$ & 0 & 0 & 0 & 0 & 0 & 0 & 29 & 100 \\
\hline Ceftriaxone(30 $\mu \mathrm{g})$ & 0 & 0 & 0 & 0 & 0 & 0 & 29 & 100 \\
\hline Cefuroxime(30 $\mu \mathrm{g})$ & 0 & 0 & 0 & 0 & 0 & 0 & 29 & 100 \\
\hline Cefoperazone/Sulbactum(5 $\mu \mathrm{g})$ & 0 & 0 & 0 & 0 & 0 & 0 & 29 & 100 \\
\hline Ciprofloxacin $(5 \mu \mathrm{g})$ & 0 & 0 & 0 & 0 & 0 & 0 & 29 & 100 \\
\hline Ertapenem(10 $\mu \mathrm{g})$ & 0 & 0 & 0 & 0 & 0 & 0 & 29 & 100 \\
\hline Gentamicin $(10 \mu \mathrm{g})$ & 0 & 0 & 0 & 0 & 4 & 13.7 & 25 & 86.2 \\
\hline Imipenem(10 $\mu \mathrm{g})$ & 0 & 0 & 0 & 0 & 0 & 0 & 29 & 100 \\
\hline Meropenem $(10 \mu \mathrm{g})$ & 0 & 0 & 0 & 0 & 0 & 0 & 29 & 100 \\
\hline Piperacillin/Tazobactum(10 $\mu \mathrm{g})$ & 0 & 0 & 0 & 0 & 4 & 13.7 & 25 & 86.2 \\
\hline 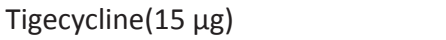 & 0 & 0 & 5 & 17.2 & 2 & 6.8 & 22 & 75.8 \\
\hline Cefepime $(30 \mu \mathrm{g})$ & 0 & 0 & 0 & 0 & 6 & 20.6 & 23 & 79.3 \\
\hline $\begin{array}{l}\text { Trimethoprim/Sulfamethoxazole } \\
(1.25 / 23.75 \mu \mathrm{g})\end{array}$ & 0 & 0 & 0 & 0 & 0 & 0 & 29 & 100 \\
\hline
\end{tabular}

Table 5. Direct antibiotic susceptibility correlation for K.pneumoniae $(n=23)$

\begin{tabular}{|c|c|c|c|c|c|c|c|c|}
\hline \multirow[t]{3}{*}{ Antibiotics used } & \multicolumn{8}{|c|}{ Direct susceptibility method } \\
\hline & \multicolumn{2}{|c|}{$\begin{array}{l}\text { Very major } \\
\text { error }\end{array}$} & \multicolumn{2}{|c|}{ Major error } & \multicolumn{2}{|c|}{$\begin{array}{l}\text { Minor error/ } \\
\text { essential } \\
\text { agreement }\end{array}$} & \multicolumn{2}{|c|}{ Concordance } \\
\hline & NO. & $\%$ & NO. & $\%$ & NO. & $\%$ & NO. & $\%$ \\
\hline Amikacin $(30 \mu \mathrm{g})$ & 0 & 0 & 0 & 0 & 4 & 17.3 & 19 & 82.6 \\
\hline Amoxicillin/Clavulanic Acid(10 $\mu \mathrm{g})$ & 1 & 4.3 & 0 & 0 & 2 & 8.6 & 20 & 86.9 \\
\hline Ampicillin $(10 \mu \mathrm{g})$ & 0 & 0 & 0 & 0 & 0 & 0 & 23 & 100 \\
\hline Ceftriaxone(30 $\mu \mathrm{g})$ & 0 & 0 & 0 & 0 & 0 & 0 & 23 & 100 \\
\hline Cefuroxime $(30 \mu \mathrm{g})$ & 0 & 0 & 0 & 0 & 0 & 0 & 23 & 100 \\
\hline Cefoperazone/Sulbactum(5 $\mu \mathrm{g})$ & 0 & 0 & 0 & 0 & 0 & 0 & 23 & 100 \\
\hline Ciprofloxacin(5 $\mu \mathrm{g})$ & 0 & 0 & 0 & 0 & 0 & 0 & 23 & 100 \\
\hline Ertapenem(10 $\mu \mathrm{g})$ & 0 & 0 & 0 & 0 & 2 & 8.6 & 21 & 91.3 \\
\hline Gentamicin(10 $\mu \mathrm{g})$ & 0 & 0 & 0 & 0 & 2 & 8.6 & 21 & 91.3 \\
\hline Imipenem(10 $\mu \mathrm{g})$ & 0 & 0 & 0 & 0 & 0 & 0 & 23 & 100 \\
\hline Meropenem(10 $\mu \mathrm{g})$ & 0 & 0 & 0 & 0 & 2 & 8.6 & 21 & 91.3 \\
\hline Piperacillin/Tazobactum(10 $\mu \mathrm{g})$ & 0 & 0 & 0 & 0 & 0 & 0 & 23 & 100 \\
\hline Tigecycline(15 $\mu \mathrm{g})$ & 0 & 0 & 1 & 4.3 & 2 & 8.6 & 20 & 86.9 \\
\hline Cefepime(30 $\mu \mathrm{g})$ & 0 & 0 & 0 & 0 & 2 & 8.6 & 21 & 91.3 \\
\hline \multicolumn{9}{|l|}{ Trimethoprim/Sulfamethoxazole } \\
\hline$(1.25 / 23.75 \mu \mathrm{g})$ & 0 & 0 & 0 & 0 & 22 & 95.6 & & \\
\hline
\end{tabular}


Table 6. Direct antibiotic susceptibility correletion for S.aureus and Coagulase Negative Staphylococcus ( $\mathrm{n}=32$ )

\begin{tabular}{|c|c|c|c|c|c|c|c|c|}
\hline \multirow[t]{3}{*}{ Antibiotics used } & \multicolumn{8}{|c|}{ Direct susceptibility method } \\
\hline & \multicolumn{2}{|c|}{$\begin{array}{l}\text { Very major } \\
\text { error }\end{array}$} & \multicolumn{2}{|c|}{ Major error } & \multicolumn{2}{|c|}{$\begin{array}{l}\text { Minor error/ } \\
\text { essential } \\
\text { agreement }\end{array}$} & \multicolumn{2}{|c|}{ Concordance } \\
\hline & NO. & $\%$ & NO. & $\%$ & NO. & $\%$ & NO. & $\%$ \\
\hline Cefoxitin $(30 \mu \mathrm{g})$ & 0 & 0 & 0 & 0 & 3 & 8.10 & 29 & 90.62 \\
\hline Clindamycin(2 $\mu \mathrm{g})$ & 1 & 2.7 & 0 & 0 & 0 & 0 & 31 & 96.87 \\
\hline Ciprofloxacin (5 $\mu \mathrm{g})$ & 0 & 0 & 0 & 0 & 0 & 0 & 32 & 100 \\
\hline Erythromycin $(5 \mu \mathrm{g})$ & 0 & 0 & 0 & 0 & 0 & 0 & 32 & 100 \\
\hline Linezolid $(30 \mu \mathrm{g})$ & 0 & 0 & 0 & 0 & 0 & 0 & 32 & 100 \\
\hline Gentamicin (30 $\mu \mathrm{g})$ & 0 & 0 & 0 & 0 & 2 & 5.4 & 30 & 93.75 \\
\hline Penicillin(10unit) & 0 & 0 & 0 & 0 & 0 & 0 & 32 & 100 \\
\hline Rifampicin(5 $\mu \mathrm{g})$ & 1 & 2.7 & 0 & 0 & 0 & 0 & 31 & 96.87 \\
\hline Teicoplanin $(30 \mu \mathrm{g})$ & 0 & 0 & 4 & 10.8 & 4 & 10.8 & 24 & 75 \\
\hline
\end{tabular}

Table 7. Direct antibiotic susceptibility correletion for Enterococcus faecium and Enterococcus faecalis $(n=5)$

\begin{tabular}{|c|c|c|c|c|c|c|c|c|}
\hline \multirow[t]{3}{*}{ Antibiotics used } & \multicolumn{8}{|c|}{ Direct susceptibility method } \\
\hline & \multicolumn{2}{|c|}{$\begin{array}{l}\text { Very major } \\
\text { error }\end{array}$} & \multicolumn{2}{|c|}{ Major error } & \multicolumn{2}{|c|}{$\begin{array}{l}\text { Minor error/ } \\
\text { essential } \\
\text { agreement }\end{array}$} & \multicolumn{2}{|c|}{ Concordance } \\
\hline & NO. & $\%$ & NO. & $\%$ & NO. & $\%$ & NO. & $\%$ \\
\hline 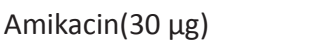 & 0 & 0 & 0 & 0 & 0 & 0 & 5 & 100 \\
\hline Ampicillin $(10 \mu \mathrm{g})$ & 0 & 0 & 0 & 0 & 0 & 0 & 5 & 100 \\
\hline \multicolumn{9}{|l|}{ High Level Gentamicin } \\
\hline \multicolumn{9}{|l|}{ High Level Streptomycin } \\
\hline 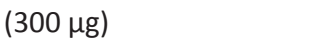 & 0 & 0 & 0 & 0 & 0 & 0 & 5 & 100 \\
\hline 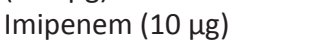 & 0 & 0 & 0 & 0 & 0 & 0 & 5 & 100 \\
\hline Meropenem $(10 \mu \mathrm{g})$ & 0 & 0 & 0 & 0 & 0 & 0 & 5 & 100 \\
\hline Penicillin (10 units) & 0 & 0 & 0 & 0 & 0 & 0 & 5 & 100 \\
\hline Teicoplanin (30 $\mu \mathrm{g})$ & 0 & 0 & 0 & 0 & 0 & 0 & 5 & 100 \\
\hline Vancomycin $(30 \mu \mathrm{g})$ & 0 & 0 & 0 & 0 & 0 & 0 & 5 & 100 \\
\hline
\end{tabular}

subcultures. Also blood cultures positive for fungus were also excluded from our study. Frequency of isolation in our study for gram negative isolates were found to be higher than gram positive isolates. This was similar to many other studies ${ }^{1,2}$. There was a contradiction in many other studies, that gram positive isolates was higher than gram negative isolates ${ }^{11,19}$. In the study done by fay and Oldfather, method of standardisation of inoculum was directly on the blood samples. Due to this there was a possibility of different inhibitory factors present in the blood to interfere with the results produced ${ }^{11}$. Therefore to avoid the error that can occur due to standardisation of inoculums we followed the guidelines of BSAC $^{6}$. For standardisation we used the method of diluting the blood samples with sterile saline, which reduced the inhibitory factor.

We used different volumes which was slightly different from the study done by Fay and Oldfather. They used an inoculum of $0.03 \mathrm{ml}$ whereas in the present study we used a volume of $5 \mathrm{ml}$ sterile water for the dilution of blood culture samples, according to BSAC guidelines ${ }^{6}$. Similar 
study done from an oncology centre in Eastern India also used the similar method instead of sterile water they used $0.45 \%$ saline ${ }^{1}$.

Since 1970's many studies have been done based on comparison of Direct susceptibility testing with that of conventional testing. Even though several studies reported an agreement of $90-97 \%$, repeating the sensitivity with the conventional method is proposed. In our study the percentage rate for very major error was found to be low followed by major error and the minor error rate was observed to be highest. Similar findings were reported in the study done by Washington and Johnson were the direct susceptibility testing was found to be both feasible and accurate as compared with standardised susceptibility testing ${ }^{12}$. Studies done by Mirret et al and Doern et al also observed the error rates to be similar to our study with however they did not report any very major errors ${ }^{13,14}$.

A study done in Eastern India also showed that the rate of errors to be less ${ }^{1}$. However, the very major error rate was found to be high in several other studies which contradict the findings in our study $^{10,15}$. Among the antibiotic panels evaluated for the gram negative isolates, it was observed that, very major errors were found in E.coli and Klebsiella for drugs amikacin , amoxicillin clavulanic acid, and trimethoprim/sulfamethoxazole and major error for amikacin, amoxicillin clavunic acid, tigecycline, and ciprofloxacin. Several other studies have reported different error rates for different antibiotic panels ${ }^{1,16,17}$. In case of gram positive isolates we obtained very major errors for clindamycin and rifampicin and major error for teicoplanin.

The frequent isolates in gram positive bacteria was S.aureus which was consistent with earlier studies ${ }^{18}$. Similarly in Gram negative isolates it was Escherichia coli followed by Klebsiella species. The isolate rate in case of gram negative bacteria was similar to the previously reported studies $^{10,18,19}$. Among gram negative bacteria, imipenem and meropenem showed lesser resistance. Enterobacteriaceae family showed high resistance to ampicillin, amoxillin and gentamicin which was also reported in the studies ${ }^{14,20}$. All the strains of $S$.aureus isolated in our study were found to be resistant to penicillin which was comparable to another study done by Garg A et al..$^{21}$.
Since there was an concordance of $94.7 \%$ for Direct susceptibility testing result obtained by gram staining as compared to that of standard susceptibility testing, we can suggest the use of DAST as a part of empirical therapy.

\section{CONCLUSION}

We compared DAST and standard AST for blood culture isolates. For gram negative bacilli, the concordance rate between these two methods was $94.14 \%$, whereas for gram positive cocci, it was $95.26 \%$. Gram negative bacilli showed highest concordance of $100 \%$ for the following antibiotics; ampicillin, cefrriaxone, cefuroxime and meropenem. Gram positive cocci showed highest concordance for clindamycin, erythromycin and pencillin. The inoculum size influences the result of DAST and standard AST. Therefore standardization of inoculum is crucial. To conclude DAST can be used to start early antibiotic treatment for bacteraemia/septicaemia.

\section{ACKNOWLEDGMENTS}

The authors would like extend sincere gratitude to the university, guide for their exemplary guidance, constant encouragement and careful monitoring throughout the study.

\section{REFERENCES}

1. Goel G, Das D, Mukherjee S, Bose S, Das K, Bhattacharya S. A method for early detection of antibiotic resistance in positive blood cultures: Experience from an oncology centre in eastern India. Indian J Med Microbiol. 2015; 33(5):53.

2. Coorevits L, Boelens J, Claeys G. Direct susceptibility testing by disk diffusion on clinical samples: a rapid and accurate tool for antibiotic stewardship. Eur J of Clin Microbiol Infect Dis. 2015; 34(6):1207-1212.

3. Synder J.W, Munier G.K, Johnson C.L. Direct Comparison of the BD Phoenix System with the MicroScanWalkAway System for Identification and Antimicrobial Susceptibility Testing of Enterobacteriaceae and Nonfermentative GramNegative Organisms. J.Clin.Microbiol. 2008; 46(7):2327-2333.

4. Battacharya S. Blood culture in India. A Proposal for a National Programme for Early detection of Sepsis. Indian J Med Microbiol. 2005; 23(4):220-6

5. Bruins JM, Bloembergen P, Rujis JHM, Wolfhagen M. Identification and Suceptibility testing of Enterobacteriaceae and Pseudomonas 
aeruginosa by Direct Inoculation from Positive BACTEC Blood culture bottles into Vitek 2. J.Clin Microbio. 2004; 42(1): 7-11

6. Mandy Wootton. BSAC standardized disc susceptibility testing method (version 12). J Antimicrob Chemother 2012: 15-16.

7. Noman F, Jehan A, Ahmed A. Reliability of Direct Sensitivity Determination of Blood Cultures. J Coll Physicians Surg Pak 2008; 18(10): 660-661.

8. Collee J, Mackie T, McCartney J. Mackie \& McCartney practical medical microbiology.New York: Churchill Livingstone; 1996:121-124.

9. CLSI. Performance Standards for Antimicrobial Susceptibility Testing; Twenty-Sixth edition. Informational Supplement. CLSI document M100-S26. Clinical and Laboratory Standards Institute; 2016.

10. De Cueto M, Ceballos E, Martinez-Martinez L, Perea E, Pascual A. Use of Positive Blood Cultures for Direct Identification and Susceptibility Testing with the Vitek 2 System. J Clin Microbiol. 2004; 42(8):3734-3738.

11. Fay D,Oldfather EJ. Standardisation of direct susceptibility test for blood Cultures. J Clin Microbiol. 1979; 9;347-50.

12. Johnson JE,Washington JA.Comparison of direct and stanmdardised antimicrobial susceptibility testing of positive blood cultures.Antimicrob Agent chemother 1976; 10: 211-4.

13. MirrettS, RellerBL. Comparision of Direct and Standard Antimicrobial Disk Susceptibility Testing for Bacteria Isolated from Blood. J Clin Microbiol.1979; 10(4):482-487.

14. Doern G, Scott D, Rashad A, Kim K. Evaluation of a direct blood culture disk diffusion antimicrobial susceptibility test. Antimicrob
Agents Chemother. 1981; 20(5):696-698

15. Haishen Kong, Shujin Zhang, Xiao Chen, Weili Zhang, Qing Yang, Yajie Fu et al. Rapid Identification and Antimicrobial Susceptibility Testing Directly from Blood Cultures of Gramnegative and Gram-positive Isolates. Clinical Laboratory. 2013; 59(01+02/2013).

16. Yu FL, Lin MH, Lee JC, Lian LY, Lin CW, Chen CT et al. Comparison of Antimicrobial Susceptibility Testing of Isolates from Blood Cultures by Direct Inoculation Method and PHOENIX. J Biomed Lab Sci. 2011; 23(1):23-27.

17. Coyle BM,Mcgonangle AL,Plorde JJ,Clausen RC,FritzDS. Rapid Antimicrobial Susceptibility Testing of Isolates from Blood Cultures by Direct Inoculation and Early Reading of Disk Diffusion Tests. J Clin Microbiol.1984; 20(3):473.

18. Karki S, Rai G, Manandhar R. Bacteriological Analysis and Antibiotic Sensitivity Pattern of Blood Culture Isolates in Kanti Children Hospital. J. Nepal Paediatr. Soc. 2010;30(2).

19. Shanthraju L, Devi G. Evaluation of direct sensitivity testing as a method for early initiation of treatment in Gram-negative sepsis. J Acad Clin Microbiolo. 2016;18(2):131.

20. Negussie A, Mulugeta G, Bedru A, Ali I, Shimeles $D$, Lema $T$ et al. Bacteriological profile and antimicrobial sensitivity pattern of blood culture isolates among septicemia-suspected children at Tikur Anbessa Specialized Hospital and Yekatit 12 Hospital, Addis Ababa, Ethiopia. Critical Care. 2013;17(Suppl 4):P11.

21. Garg A, Anupurba S, Garg J.Goyal RK,Sen MR.Bacteriological profile and Antimicrobial Resistance of Blood culture isolates from a University Hospital.JIACM.2007;8(2):139-143. 